\title{
Primer registro de Blechnum appendiculatum (Pteridophyta: Blechnaceae) para Nuevo León, México
}

\author{
First record of Blechnum appendiculatum (Pteridophyta: Blechnaceae) for the state of Nuevo \\ León, México.
}

\author{
Carlos Gerardo Velazco Macías ${ }^{1 *}$, Glafiro José Alanís Flores² y Marco Antonio Alvarado Vázquez² \\ ${ }^{1}$ Parques y Vida Silvestre de Nuevo León. Avenida Eugenio Garza Sada \# 6604, Col. Lagos del Bosque, 64890 Monterrey, Nuevo León, México. \\ ${ }^{2}$ Facultad de Ciencias Biológicas, Universidad Autónoma de Nuevo León, 66450 San Nicolás de los Garza, Nuevo León, México. \\ *Correspondencia: carlos.velazco@gmail.com
}

\begin{abstract}
Resumen. Se registra Blechnum appendiculatum Willd. por primera vez en Nuevo León. Es éste el primer registro del género para ese estado. La especie se encontró dentro del Área Natural Protegida Estatal Sierra Cerro de la Silla, municipio de Cadereyta Jiménez; tanto el género como la especie son primer registro para el estado.
\end{abstract}

Palabras clave: flora, biodiversidad, helechos.

\begin{abstract}
Blechnum appendiculatum Willd. is first recorded in the state of Nuevo León, within the natural protected area of Sierra Cerro de la Silla, in the Cadereyta Jiménez municipality; this is first record for both genus and species in the state.
\end{abstract}

Key words: flora, biodiversity, ferns.

En el estado de Nuevo León, en el noreste de México, se han desarrollado diversos trabajos encaminados al conocimiento de la pteridoflora; destacan de manera especial los realizados en los municipios de General Zaragoza e Iturbide para la zona sur del estado (Favela, 1983 y Espinoza, 1986), en los municipios de Santiago, Garza García, Monterrey, Santa Catarina, Guadalupe, Allende, García y Abasolo para la zona centro-oeste (Rodríguez, 1987 y Ortega, 2002), y 2 trabajos más para todo el estado (Aguirre, 1983 y Aguirre y Arreguín, 1988).

Mickel y Smith (2004) registran 122 especies en el estado, mientras que estudios florísticos más recientes indican la presencia de entre 90 y 134 especies de helechos y plantas afines (Villarreal y Estrada, 2008 y Velazco, 2009). A pesar de los esfuerzos de colecta y documentación florística, el género Blechnum no había sido previamente colectado o registrado para el estado; Blechnum appendiculatum, en particular, presenta una amplia distribución en México en los estados de Chiapas, Colima, Distrito Federal, Guerrero, Hidalgo, Jalisco, estado de México, Michoacán, Morelos, Nayarit, Oaxaca,

Recibido: 15 abril 2010; aceptado: 03 agosto 2010
Puebla, Querétaro, San Luis Potosí, Tamaulipas, Tlaxcala y Veracruz, extendiéndose en su distribución hacia Centro y Sudamérica (Mickel y Smith, 2004), donde habita en cortes de carreteras y cañadas, en bosques húmedos de montaña y bosques de pino-encino.

Durante los recorridos de rutina para la vigilancia del Área Natural Protegida Estatal Sierra Cerro de la Silla, se detectó la presencia de un helecho poco común y con una distribución muy puntual en una cañada denominada Las Boquillas, entre los límites de los municipios de Santiago y Cadereyta Jiménez (Fig. 1), entre los 600 y 800 m snm, del cual se colectaron muestras botánicas para su posterior tratamiento e identificación. Al utilizar las claves taxonómicas de Mickel y Smith (2004), se identificó como una especie del género Blechnum, de manera específica $B$. appendiculatum, que resultó ser el primer registro para el estado (Fig. 2).

La especie se desarrolla bajo un clima de tipo templado semicálido subhúmedo con lluvias en verano, $(\mathrm{A}) \mathrm{C}\left(\mathrm{W}_{0}\right)$ y $(A) C\left(W_{1}\right)$, con un porcentaje de lluvia invernal entre 5 y $10.2 \mathrm{~mm}$, la temperatura media anual es de $18^{\circ} \mathrm{C}$ y la precipitación del mes más seco es menor de $40 \mathrm{~mm}$, según la clasificación de Köppen modificado por García (SPP, 1980). La geología de la zona es de origen sedimentario, 


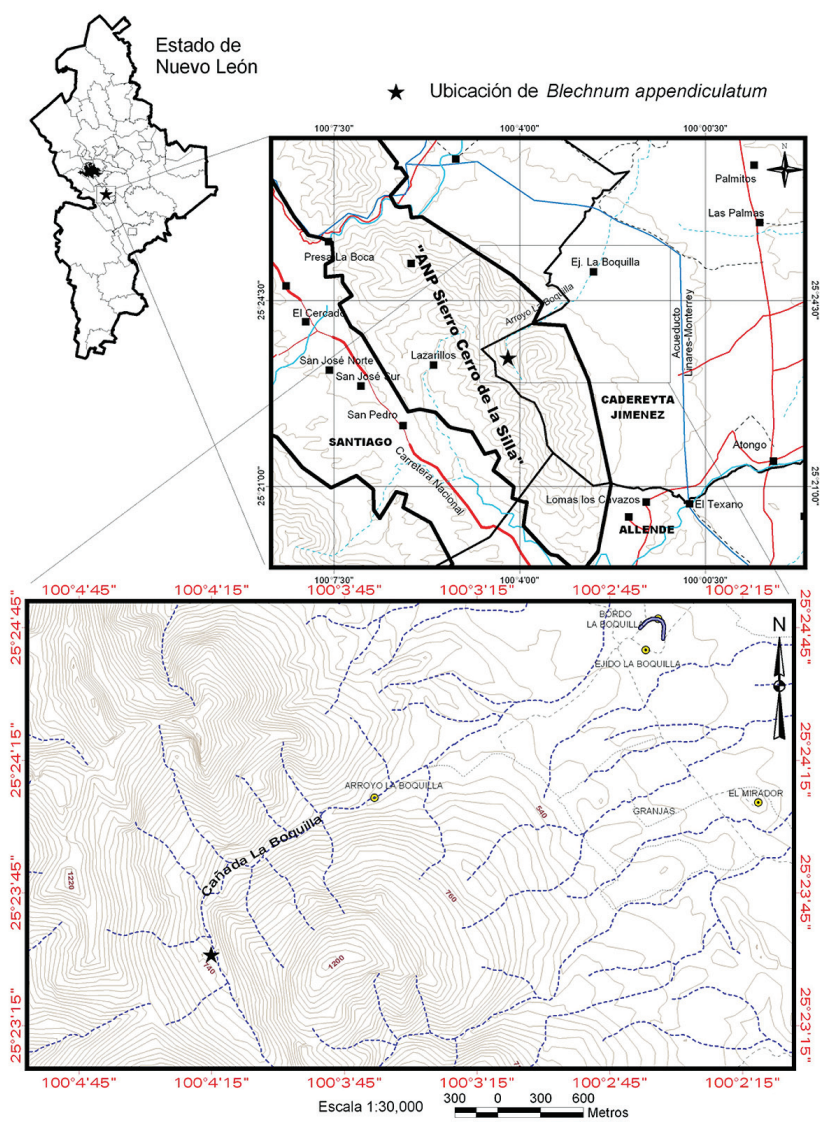

Figura 1. Localización de Blechnum appendiculatum en el estado de Nuevo León, México. con rocas de tipo caliza; en algunas zonas se mezcla la caliza y la lutita (CETENAL, 1977a). Se presentan suelos de tipo litosol más rendzina con textura media en los $30 \mathrm{~cm}$ superficiales del suelo (CETENAL, 1977b).

De acuerdo con CETENAL (1977c), se presenta vegetación de matorral subinerme e inerme, así como boque de encino (Quercus spp.) mezclado con el matorral, aunque de manera particular y de acuerdo con lo observado en campo, $B$. appendiculatum habita en bosque mixto de Platanus rzedowskii, Sargentia greggii y Quercus spp.; se presentan además las siguientes especies de helechos: Adiantum capillus-veneris, Pteris cretica, Pteris vittata, Thelypteris ovata var. lindheimeri, Thelypteris concinna, Dennstaedtia globulifera, Polypodium plesiosorum, Polypodium polypodioides var. michauxianum, Tectaria heracleifolia, Phanerophlebia umbonata, Cheilanthes aemula, Cheilanthes alabamensis y Llavea cordifolia; otras especies herbáceas que destacan en el sotobosque son Stellaria cuspidata, Ranunculus petiolaris, Rivina humilis y Tradescantia pringlei.

Ejemplares de respaldo. MÉXICO, Nuevo León, municipio de Cadereyta Jiménez: Cañada Las Boquillas, límite con el municipio de Santiago, 700 m snm, 25'23'38.97’N y -10004'17.10”O, 21. I. 2010, Velazco Macías C.G. (ejemplares de herbario y fotografías digitales), UNL.

Esta adición a la flora de Nuevo León es un ejemplo más de la importancia de mantener la exploración botánica en el estado y, desde el punto de vista de la conservación,

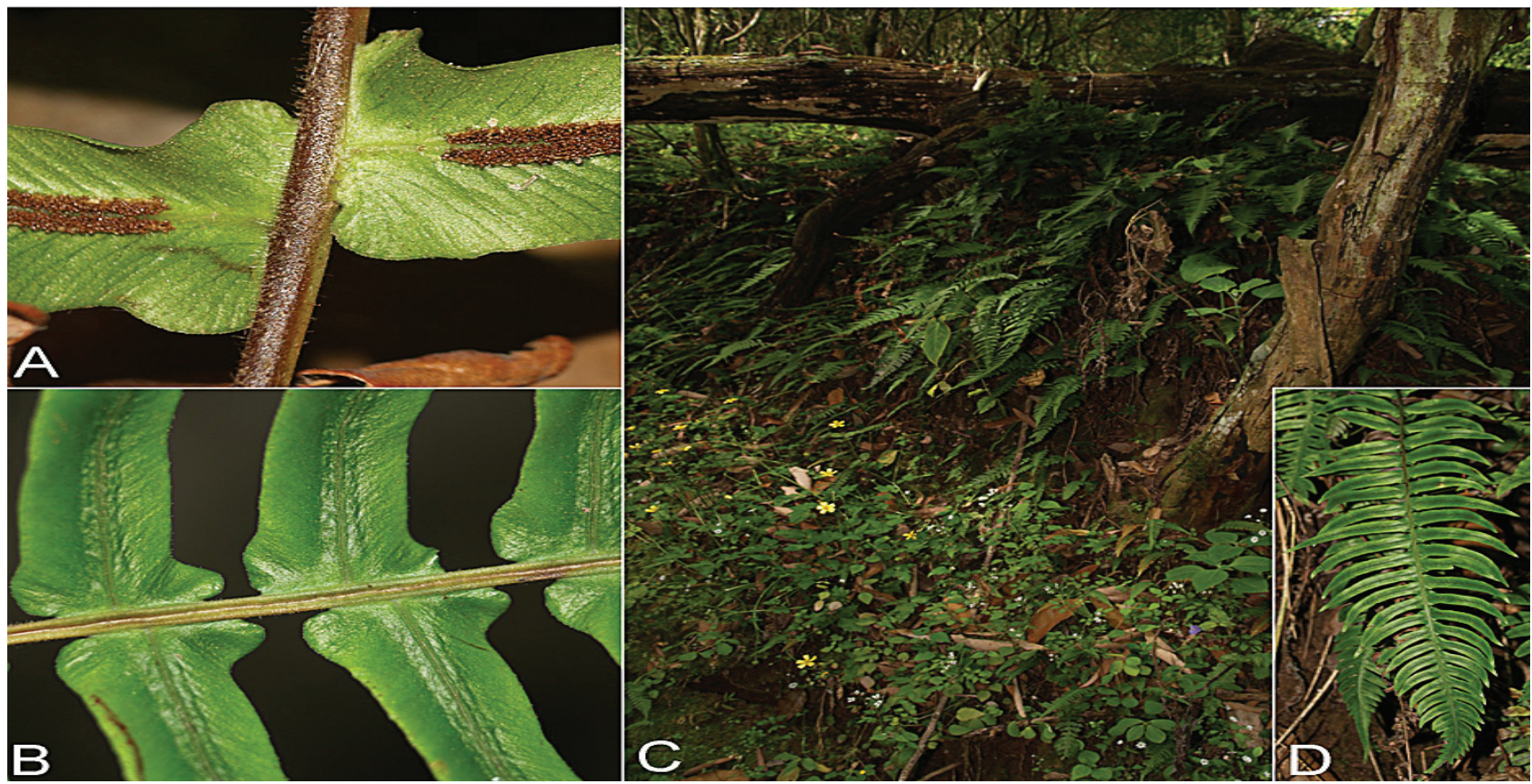

Figura 2. A, vista abaxial de las pinnas basales donde se aprecia la vellosidad característica de la especie; B, vista adaxial de las pinnas basales y el raquis acanalado; $\mathrm{C}$, vista del hábitat; D, vista general de la fronda de Blechnm appendiculatum. 
se da un mayor peso al área natural protegida estatal como un reducto de ecosistemas y especies únicas en el estado.

Se agradece al Biól. Héctor Villalón Moreno y al Ing. Jorge Alberto Martínez Pineda por su apoyo en la realización del trabajo de campo y la elaboración de la cartografía.

\section{Literatura citada}

Aguirre, C. R. 1983. Contribución al conocimiento de la pteridoflora del estado de Nuevo León, México. Tesis, Facultad de Ciencias Biológicas, Universidad Autónoma de Nuevo León. Monterrey, N. L. 187 p.

Aguirre, C. R. y S. M. de la L. Arreguín, 1988. Claves de familias, géneros, especies y variedades de Pteridofitas del estado de Nuevo León, México. Anales de la Escuela Nacional de Ciencias Biológicas 32:9-61

CETENAL (Comisión de Estudios del Territorio Nacional). 1977a. Carta geológica. Allende G14C36. Escala 1:50,000. Instituto Nacional de Estadística, Geografía e Informática, Aguascalientes, Aguascalientes.

CETENAL (Comisión de Estudios del Territorio Nacional). 1977b. Carta edafológica. Allende G14C36. Escala 1:50,000. Instituto Nacional de Estadística, Geografía e Informática, Aguascalientes, Aguascalientes.

CETENAL (Comisión de Estudios del Territorio Nacional). 1977c. Carta de uso de suelo. Allende G14C36. Escala 1:50,000. Secretaría de Programación y Presupuesto, México, D.F.

Espinoza, O. N. 1986. Pteridoflora del municipio de Iturbide,
Nuevo León, México. Tesis, Facultad de Ciencias Biológicas, Universidad Autónoma de Nuevo León, Monterrey, $150 \mathrm{p}$.

Favela, L. S. 1983. Estudio de la flora pteridológica del municipio de General Zaragoza, Nuevo León, México. Tesis, Facultad de Ciencias Biológicas, Universidad Autónoma de Nuevo León, Monterrey. 82 p.

Mickel, J. T. y A. R. Smith. 2004. The pteridophytes of Mexico, vol. 88. The New York Botanical Garden.1055 p.

Rodríguez, L. R. 1987. Conocimiento actual de las pteridofitas en el estado de Nuevo León, México, con énfasis en la zona montañosa del centro-oeste, su taxonomía y distribución. Tesis, Facultad de Ciencias Biológicas, Universidad Autónoma de Nuevo León, Monterrey. 105 p.

SPP (Secretaría de Programación y Presupuesto). 1980. Carta estatal, Climas, Nuevo León, México. Escala 1:1,000,000. Coordinación general de los servicios nacionales de estadística, geografía e informática. México, D.F.

Ortega, H. E. 2002. Diversidad de helechos (Polypodiophyta) de Abasolo, Nuevo León, México. Tesis, Facultad de Ciencias Biológicas, Universidad Autónoma de Nuevo León. Monterrey. 93 p.

Velazco Macías, C. G. 2009. Flora del estado de Nuevo León: diversidad y análisis espacio-temporal. Tesis, doctorado. Facultad de Ciencias Biológicas, Universidad Autónoma de Nuevo León, Monterrey. 272 p.

Villarreal, Q. J. A. y E. Estrada Castillón. 2008. Listados florísticos de México. XXIV. Flora de Nuevo León. Instituto de Biología, Universidad Nacional Autónoma de México. México, D.F. 153 p. 
